\title{
REPRESENTATION OF ABERRATION DIFFRACTION EFFECTS BY MEANS OF ROTATING SECTORS
}

\author{
By A. H. Bennett
}

ABSTRACT

This paper describes a set of models constructed for the purpose of illustrating the combined effect of spherical aberration and out-of-focus conditions on the axial star image formed by any lens system of circular aperture. Representation of the star disks is accomplished by means of properly shaped rotating sectors. Figures are given showing sectors corresponding to cases in which the spherical aberration varies in amount from $1 / 8 \lambda$ to $1 / 2 \lambda$. The effect of a change of focus in the presence of spherical aberration is illustrated.

\section{CONTENTS}

I. Introduction

II. Theory _.

III. Construction of the models _._. 394

IV. Interpretation of the models _... 395

V. Summary and conclusions._. 397

\section{INTRODUCTION}

In connection with the Michelson meeting of the Optical Society of America, held in November, 1928, at Washington, the writer exhibited a set of models, illustrating certain appearances of axial star images in the focal plane of an optical system of circular aperture, in which various amounts of spherical aberration were assumed to be present. In these models each star disk chosen for illustration is represented by a rotating sector such as is commonly used to obtain a symmetrical radial distribution of brightness. ${ }^{1}$ These models were constructed for the primary purpose of presenting directly to the eye the combined effects of spherical aberration and out-of-focus conditions in displacing light from the central maximum of the diffraction pattern into the surrounding ring system of the star image.

It is well recognized that a study of the resultant effect of aberrations upon the diffraction pattern at the focus of any lens system requires a consideration of the wave theory of light and is of fundamental importance in determining the character of compensation that

1 The application of the rotating sector in representing the image disk of a planet as seen through an ideal aberration-free telescope has been described by H. Seeliger, Bay. Akad. d. Wiss. Munchen, Sitzb. Math-Phys. Cl., 19, p. 404; 1896-1899. 
has been attained in design and construction. Accordingly, for use in such investigations Rayleigh, ${ }^{2}$ Conrady, ${ }^{3}$ Buxton, ${ }^{4}$ and Stewart ${ }^{5}$ have deduced methods for evaluating the intensity distribution in the star image at and in the vicinity of the focus of a lens when the emergent wave front is distorted by the presence of aberration.

The set of models is of considerable interest in studying the formation and quality of images produced by optical systems because it affords a means for representing in concrete form the different intensity distributions in the diffraction patterns which result from selected values of the several geometric aberrations, together with given out-of-focus conditions. But it is believed that the models have a more general use in furnishing a means by which aberration diffraction effects, and the limitations thereby imposed on the definition, can be presented in tangible form to classes in physics. For this reason plates of the sectors have been reproduced from which it is a relatively simple matter to construct a set of models for the cases illustrated.

\section{THEORY}

The problem of determining the intensity distribution in the image of an axial point source in the focal plane of a lens having a circular aperture was first considered by Airy, ${ }^{6}$ who showed that, with an ideally perfect objective lens entirely free from aberration, the distance between the central maximum and the first dark ring in the diffraction pattern subtends an angle of $\frac{1.22 \lambda}{2 r}$ radians at the objective, where $\lambda$ is the wave length and $r$ is the semiaperture of the lens.

Dawes, ${ }^{7}$ in his measurements on double stars, experimentally showed that if two stars are to be resolved the minimum angular separation must be such that the central maximum of one image falls on the first dark ring of the other.

In practice, it is of importance to know the effect of the presence of aberrations on the definition produced by a lens or, in other words, the effect produced by distortion of an emergent wave front from its ideally spherical form. ${ }^{8}$ Accordingly, Rayleigh in an extension of Airy's work considered the case of a lens possessing second-order longitudinal spherical aberration and concluded that, if the aberration introduces a phase difference of $1 / 4 \lambda$ between the axial and marginal portions of the emergent wave front on arrival at the paraxial focal point, the definition of an object point is just noticeably impaired.

\footnotetext{
2 Lord Rayleigh, Phil. Mag., 8, pp. 403-411; 1879.

${ }^{3}$ A. E. Conrady, M. N. R. A. S., 79, pp. 575-593; 1918-19.

1 Arnold Buxton, M. N. R. A. S., 81, pp. 547-565; 1920-21.

${ }^{5}$ G. C. Stewart, Trans. Roy. Soc., London, Series A, 225, pp. 131-198; 1926.

6 G. B. Airy, Camb. Phil. Trans.; 1834.

7 W. R. Dawes, Mem. Roy. Ast. Soc., 35, p. 158; 1865.

8 In order to determine the exact effect of various amounts of spherical aberration on resolving power, a miniature set of sectors, such as are described in this paper, has been constructed by the writer.
} 
Conrady and later Buxton have extended the study of aberration diffraction effects to include all forms of spherical aberration. In addition to secondary longitudinal aberration, the higher orders were introduced and also the effects of a change of focus combined with the aberration were given consideration, since in most cases in the presence of aberration the paraxial focus is not the most favorable for producing greatest condensation of light in the central maximum of the star image. If one knows the total phase difference arising from change of focus, and from each of the several orders of spherical aberration, the intensity distribution in the star image can be computed.

By means of a trigonometric tracing of rays through a projected lens system, or by application of the Hartmann test to an actual lens, the longitudinal spherical aberration can be determined. Martin ${ }^{9}$ has dealt with the method of transforming longitudinal spherical aberration to phase difference at any selected focus, and this transformation is in most cases the first step to be taken in determining the intensity distribution in axial images of point sources formed by any particular lens or system of lenses. Longitudinal spherical aberration can be expressed in the form

$$
L=A_{1} r^{2}+A_{2} r^{4}+A_{3} r^{6}, \text { etc. }
$$

where $A_{1}, A_{2}, A_{3}$, etc., are the constants for the longitudinal spherical aberration of the several different orders and $r$ is the semiaperture. The phase aberration at the paraxial focus is

$$
\eta=\frac{2 \pi}{\lambda f^{2}} \int_{0}^{r} L r d r
$$

where $f$ is the image distance. At an axial point distant $m$ from the paraxial focus the phase aberration is

$$
\eta=-\frac{\pi m r^{2}}{\lambda f^{2}}+\frac{2 \pi}{\lambda f^{2}} \int_{0}^{r} L r d r
$$

The first term of the right-hand member of equation (3) represents the effect of displacing the focus to an axial point other than the paraxial focus, and it furthermore shows that by selection of a displacement, $m$, of suitable sign and magnitude the total phase difference for any particular case can be made a minimum.

Equation (3) can be written

$$
\eta=\varphi_{1} r^{2}+\varphi_{2} r^{4}+\varphi_{3} r^{6}, \text { etc. }
$$

in which the term in $r^{2}$ represents the effect of a change of focus, and the terms in higher even powers of $r$ represent successively increasing orders of spherical aberration. 
The intensity at any point in the star image has been expressed by Buxton in terms of the quantities of equation (4), the dimensions of the lens, and the position of the point under consideration, as

$$
\begin{aligned}
I & =\left[\int_{0}^{1} J_{o}\left(\frac{r x}{f}\right) \cos \left(\varphi_{1} r^{2}+\varphi_{2} r^{4}+\ldots\right) d r^{2}\right]^{2} \\
& +\left[\int_{0}^{1} J_{o}\left(\frac{r x}{f}\right) \sin \left(\varphi_{1} r^{2}+\varphi_{2} r^{4}+\ldots\right) d r^{2}\right]^{2}
\end{aligned}
$$

where $r$ is the semiaperture; $x$, the radial distance of the point in the star disk from the axis; and $f$, the focal length of the lens. The intensity, $I$, can be computed by mechanical quadratures with the aid of tables given by Conrady for this purpose. In computing the intensities represented in the models, an extension of these tables was necessary, as it was desired to determine the intensity at points beyond the second bright ring in the star disk.

By the method outlined above the intensity distributions in several star disks, under various conditions of focus and in the presence of different amounts of spherical aberration, were determined.

\section{CONSTRUCTION OF THE MODELS}

After determining the intensity distribution for any particular combination of aberration and focus it is necessary to compute the outline of a sector which when rotated produces the desired distribution. Such a sector must be shaped so that at any radial distance from the center of the rotating disk the total angular width of the sectored portion is proportional to the intensity to be reproduced. This condition can be fulfilled by using any number of sectors on the same disk. A multisectored disk has the obvious advantage of requiring less speed of rotation for the avoidance of flicker than does a disk having but one sector. But, in order to reproduce relatively low intensities, it is necessary that the sectored portions be correspondingly narrow; and, with a multisectored disk, the individual sectors may become so narrow that it is difficult to avoid drawing them too wide in the regions where it is necessary to represent a brightness which approaches zero. By using two sectors on each disk, as shown in Figures 4, 5, 6, 7, 8, and 9, it is not difficult to attain the necessary speed of rotation for the avoidance of flicker, and at the same time the sectors do not become unduly narrow. It is mainly a matter of choice whether the sector is bounded on one side by a straight line radial to the disk or whether it is symmetrical about a radius of the disk. However, it was decided to bound the sectors by suitably shaped symmetrical curves because by so doing there is an increased probability of averaging out the accidental errors which occur in plotting and drawing the curved boundaries. 


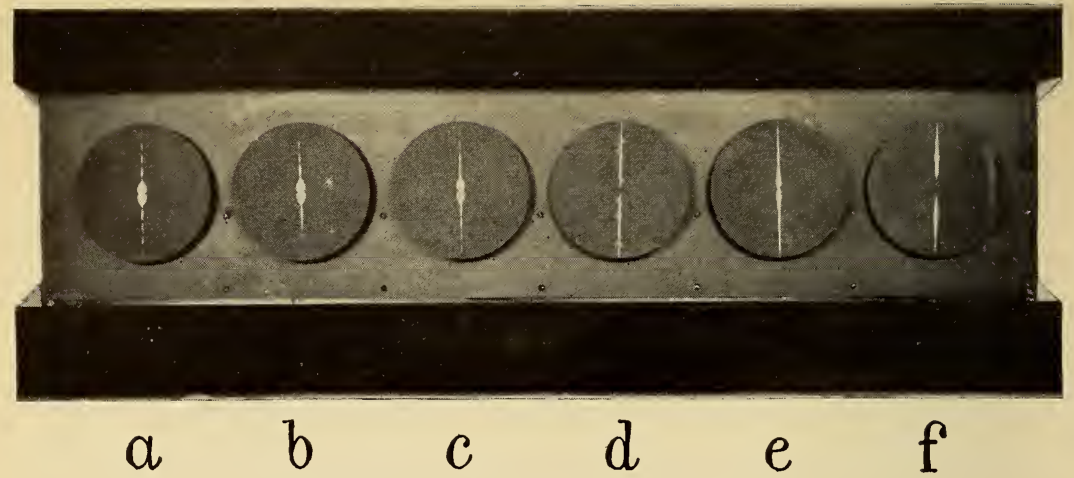

Figure 1.-Front elevation of apparatus

The sectors when rotated simulate star disks formed by lenses having various amounts of spherical aberration.

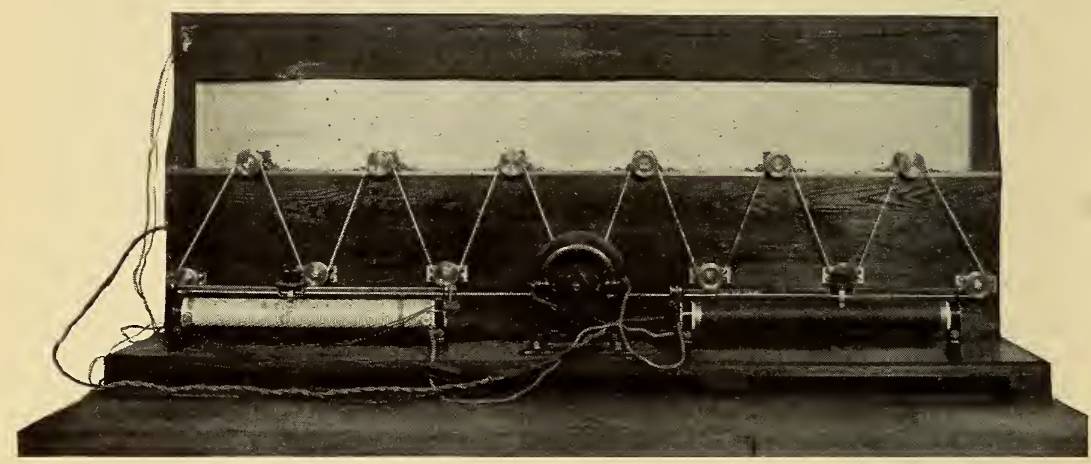

FIGURE 2.-Rear elevation of apparatus showing method of driving the sectors

By means of one long belt a single motor is used to rotate the six sectors simultaneously.

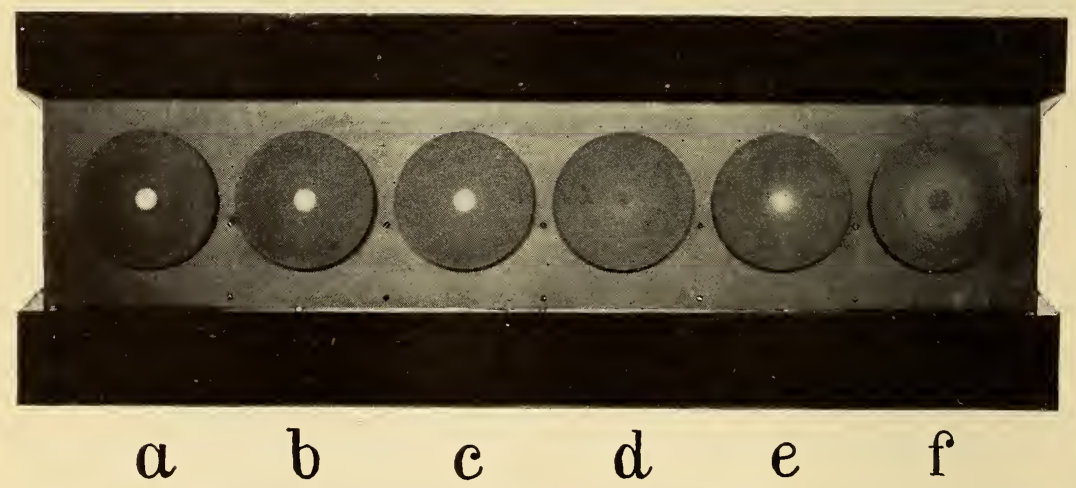

FiguRE 3.-Front elevation of apparatus showing sectors in rotation

The sectors produce brightness distributions corresponding to the following cases: (a) Diffraction pattern for a perfect image, $(b)$ diffraction pattern in the presence of $1 / 8 \lambda$ aberration, $(c)$ diffraction pattern in the presence of $1 / 4 \lambda$ aberration (Rayleigh limit), $(d)$ diffraction pattern in the presence of $1 / 2 \lambda$ aberration with image plane displaced from paraxial focus in the direction from the caustic, $(e)$ diffraction pattern in the presence of $1 / 2 \lambda$ aberration with image plane at paraxial focus, $(f)$ diffraction pattern in the presence of $1 / 2 \lambda$ aberration with image plane displaced from paraxial focus in the direction toward the caustic. 
In computing the intensities at the different points in the diffraction pattern by the method to which reference has been made the results are expressed in terms of the intensity of the central maximum of the ideal Airy disk. It is convenient to assign to this latter intensity a value of 100 per cent because it is the maximum relative intensity necessary to be reproduced by the sectors. With the intensities $I$ so expressed, and with the assumption that the rotating disk contains two symmetrical sectors, at a distance $r$ from the center of the disk the position of a point on the boundary of the sector can be expressed as

$$
\begin{aligned}
& x=r \sin \left(I \times 90^{\circ}\right) \\
& y=r \cos \left(I \times 90^{\circ}\right)
\end{aligned}
$$

the origin of the Cartesian coordinates being taken at the center of the disk.

For each of the cases illustrated in this paper a large-scale drawing: was first made showing the sectors blackened. The finished disk is simply a photographically reduced negative print of the original drawing.

The sectors were mounted on the apparatus shown in Figures 1 and 2. The photographic print containing the sector was first mounted on a cardboard disk by means of mounting tissue. This, in turn, was attached (with hard wax) to a thin, flat brass disk. The sectors were mounted as described in order to give them sufficient flatness and rigidity to assure stability and freedom from vibration when rotated at high speeds. Each disk was mounted on a rotating spindle, with provision for adjusting the disk in its own plane so that the center of the figure accurately coincides with the center of rotation. The spindles project through a large blackened sheet of aluminum which provides a suitable background for the rotating sectors. Illumination is obtained by means of a number of automobile headlight bulbs in housings above and below the sectors.

\section{INTERPRETATION OF THE MODELS}

In Figure 3, a, the perfect Airy star disk is illustrated and Figure 4 is the corresponding sector. As has been implied, the central maximum of this disk is considered as having an intensity of 100 per cent, whereas at the first dark ring the intensity decreases to zero. In the model it is, of course, impossible to avoid some reflection of light from the black portion of the disk, and consequently this ideal zero value is not quite attained. The effect of this scattered light is small, however, in comparison to the aberration diffraction effects which it is desired to represent and has been neglected in computing the shape of the sectors. The first bright ring has a maximum intensity of 1.6 per cent, while the intensity of the second bright ring 
is only 0.4 per cent. As is shown later, the presence of aberration changes these intensities by amounts which are quite appreciable.

Figure $3, b$ (sector shown in fig. 5), represents the star disk at the paraxial focus of a lens which at that point imposes $1 / 8 \lambda$ phase difference between the paraxial and marginal portions of the wave front emergent from the lens. This phase aberration of $1 / 8 \lambda$ is half of the Rayleigh limit and is considered as producing no visibly deleterious effect on the star image formed by a lens. It is of interest to note the alteration in intensities in the star disk which this small amount of aberration introduces. The intensity of the central maximum is reduced to 95 per cent, the minimum intensity in the first dark ring is no longer zero but about 0.9 per cent, while the intensity of the first bright ring is increased to 1.8 per cent. Thus, the effect of the aberration is to decrease the intensity of the central maximum and to displace this light into the surrounding pattern. The effect is very slight, however, and it is very difficult in viewing the models to detect any difference between the contrast conditions in the perfect disk (fig. $3, a$ ) and those of the $1 / 8 \lambda$ disk. This is in accord with the current idea that this amount of aberration can be tolerated in instruments of high quality.

In Figure 3, $c$, and also in the sector of Figure 6, the aberration has been increased to $1 / 4 \lambda$ which, according to Rayleigh, "begins to be decidedly prejudicial" to the definition. The intensity of the central maximum is now reduced to 80 per cent, with a further increase of illumination in the ring system and a consequent loss of contrast. Conrady suggests that this loss of contrast necessitates a more exacting tolerance of $1 / 6$ or $1 / 8 \lambda$ in instruments of the highest quality.

The effect of spherical aberration of $1 / 2 \lambda$ in magnitude is shown in Figure $3, e$, and the corresponding sector appears in Figure 7 . Here the loss of contrast in the pattern is very pronounced, and a comparison with the ideal pattern Figure $3, a$, reveals a pronounced deterioration of the quality of the image. On detail possessing sufficient contrast this amount of aberration does not, however, according to Conrady, ${ }^{10}$ seriously affect the resolving power of a lens because the central condensation remains of about the same size as those of star images produced by lenses having much smaller amounts of aberration. Thus, such a lens might resolve double stars satisfactorily and yet not be suitable for resolving planetary or lunar details in which the contrast is weak.

Disks $(d),(e)$, and $(f)$ of Figure 3 and sectors of Figures 7, 8, and 9 compose a series designed to show the result of change of focus in the presence of spherical aberration, and they also serve to illustrate

${ }_{10}$ Dictionary of Applied Physics (London), 4, p. 221; 1923. 

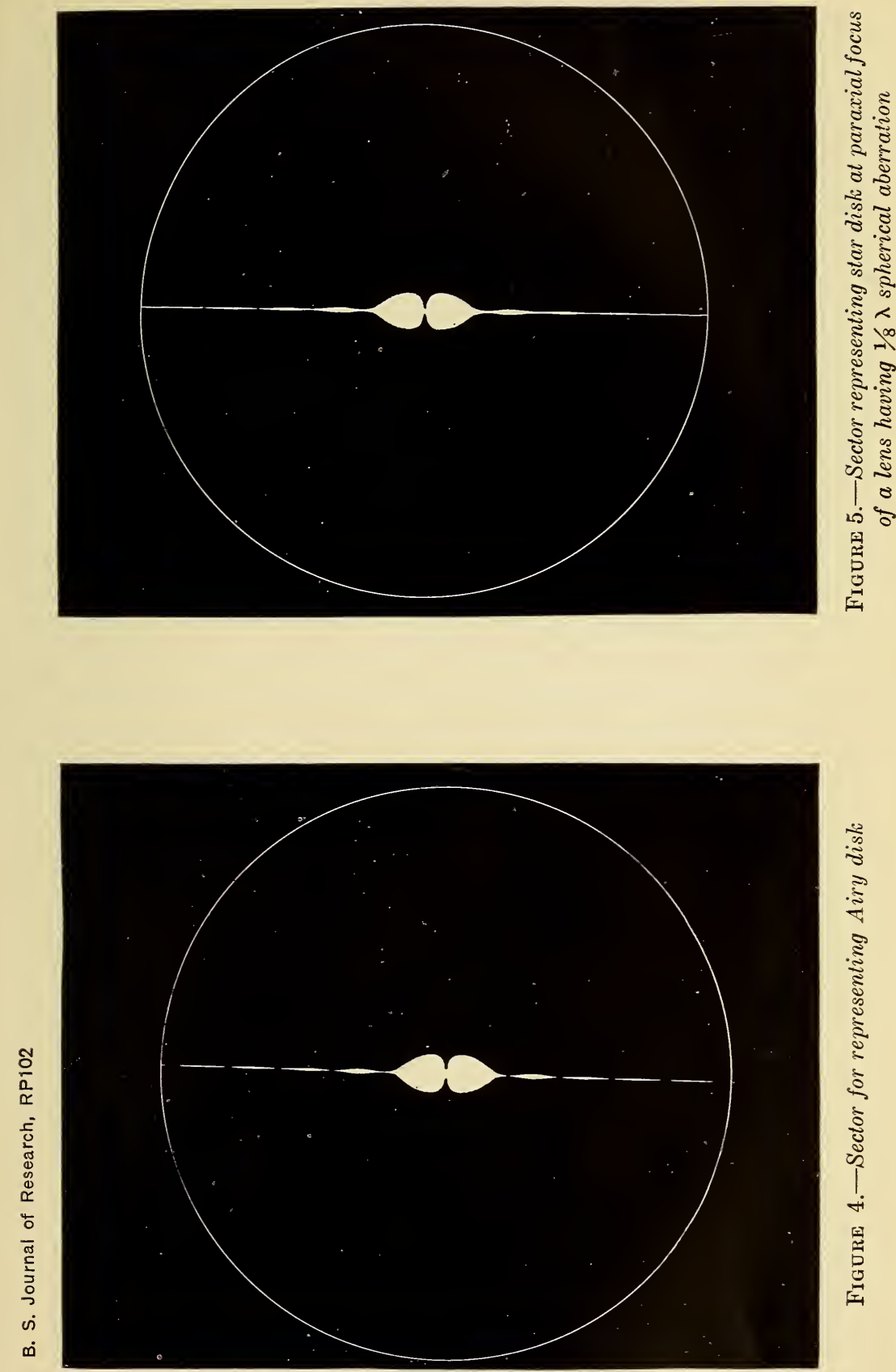

396-1 

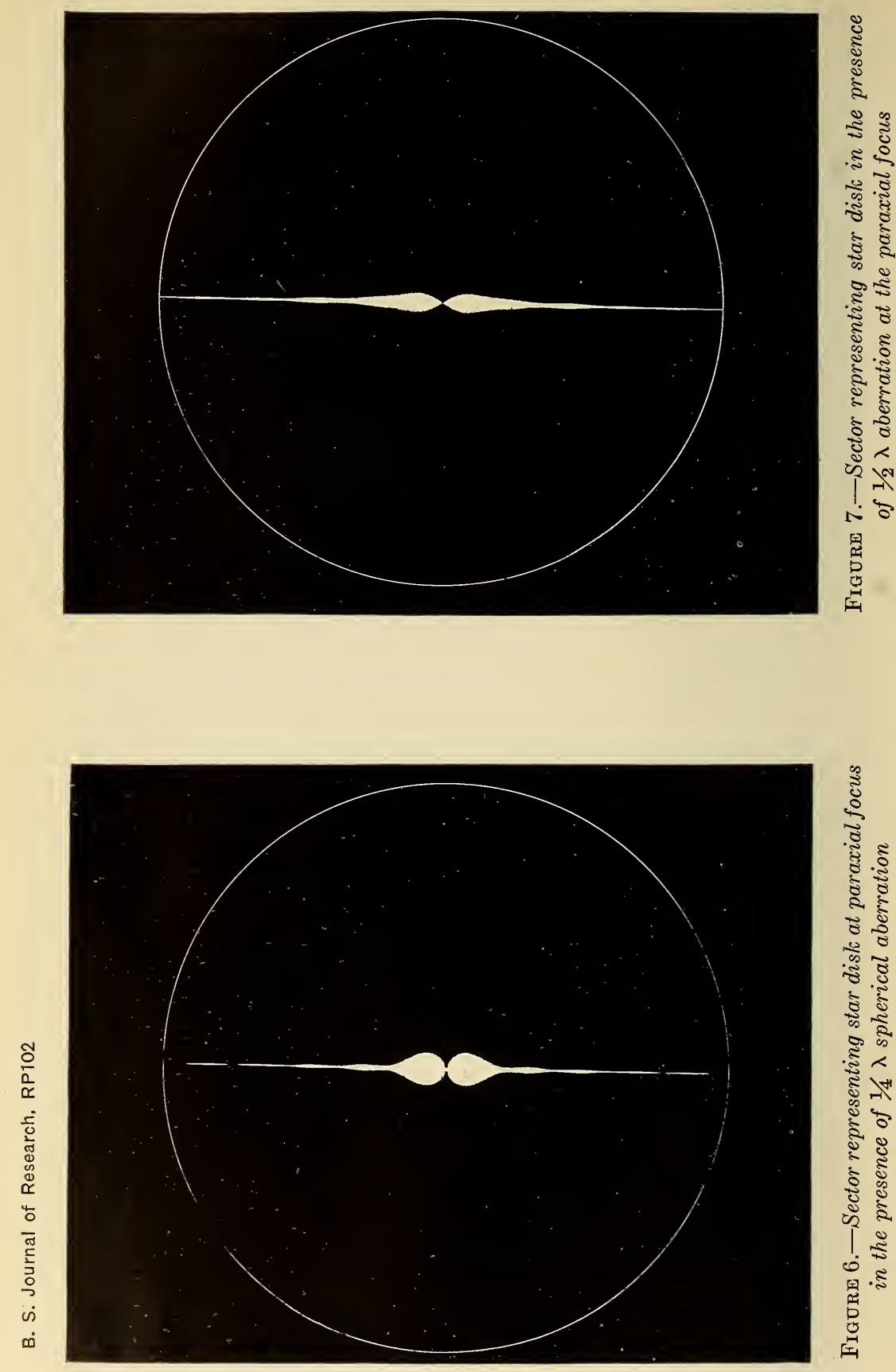

$396-2$ 

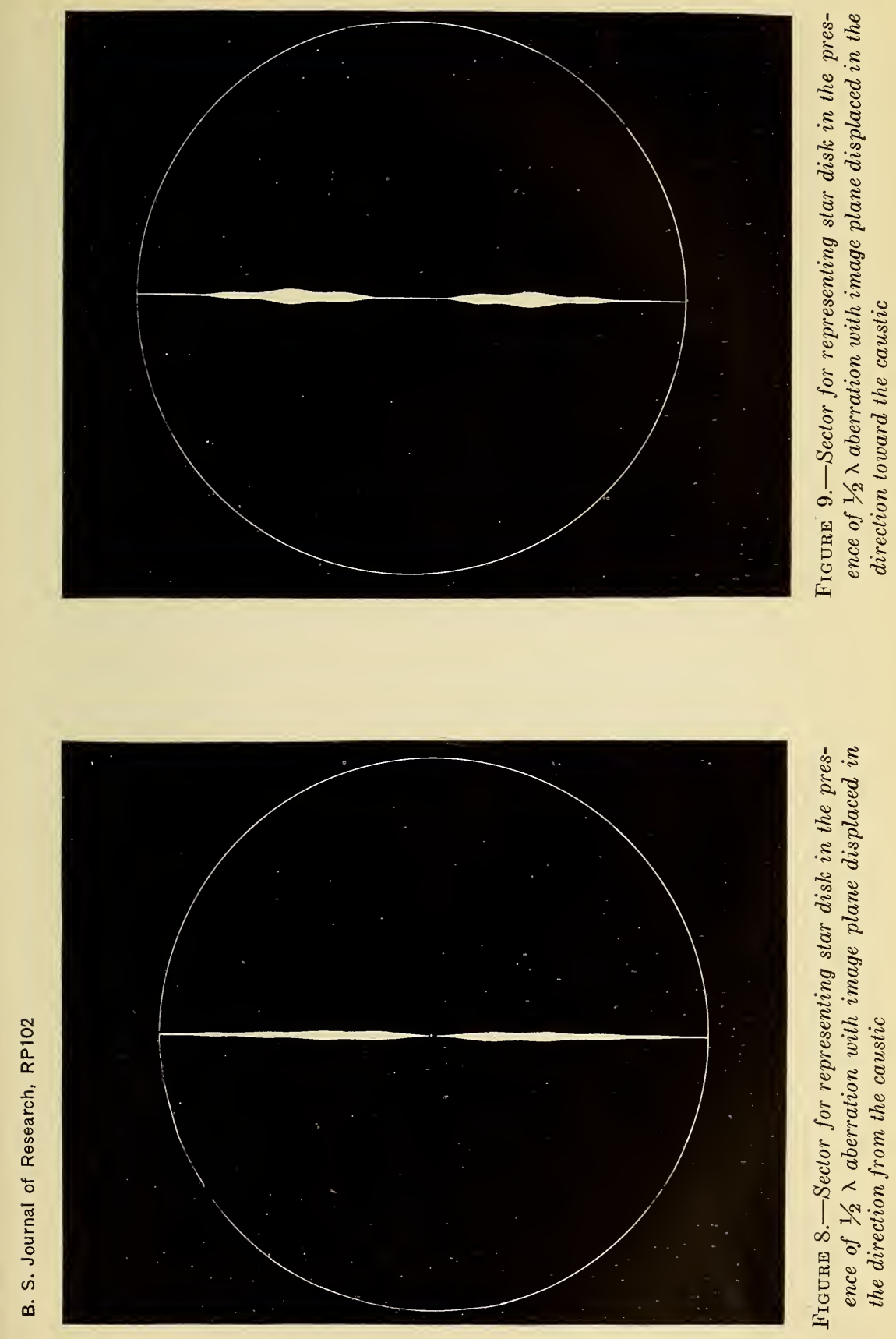

$396-3$ 
an important method ${ }^{11}$ of inspecting lenses for the presence of spherical aberration. If this defect is present, the rays, after refraction through a lens, do not pass through a single axial point but form the envelope of a surface called the caustic. If the caustic lies between the paraxial focus and the lens, the latter is under-corrected for spherical aberration, whereas in case of overcorrection the caustic is farther from the lens than is the paraxial focus. As a result of these conditions two cross sections of an emergent bundle of refracted rays, if taken to include axial points on opposite sides of the best focus, have characteristic appearances which are especially pronounced even in the presence of minute amounts of aberration.

Such appearances furnish a basis for the above-mentioned method of lens inspection which has not only the merit of high sensitivity but also that of convenience and simplicity. Let it be assumed that the star image formed by the lens is viewed through an eyepiece, as is the case with a telescope or microscope. If the eyepiece is racked from the best focus in a direction such that the cross section of the emergent bundle cuts the caustic, the out-of-focus star image is seen to consist of a sharply defined system of interference fringes. But if the eyepiece is racked away from the caustic the rings are very indistinct or even invisible.

For the case of the previously described star image of Figure 3,e, intensities were also computed for out-of-focus conditions at two positions along the axis on opposite sides of the paraxial focus. In each instance the displacement from the paraxial focus was such that the phase difference at the selected focus was $1 \lambda$, while, as previously mentioned, at the paraxial focus the phase difference is $1 / 2 \lambda$. As a result the model shown in Figure $3, f$, represents the image of Figure $3, e$, when the latter is displaced from the paraxial focus in the direction of the caustic, while $d$ represents the extrafocal image on the opposite side of the paraxial focus. An inspection of the sectors (figs. 8 and 9 , respectively) shows that the contrast is stronger in the image displaced toward the caustic. This is easily noticeable when viewing the actual models; and, although it is hardly possible to reproduce photographically the small variations of contrast in the rotating sectors, the illustrations (fig. $3, d$ and $f$ ) show some difference in the appearance of these two out-of-focus images.

\section{SUMMARY AND CONCLUSIONS}

1. By means of models such as are described it is possible to represent directly to the eye the intensity distribution produced in a monochromatic axial star image formed by any actual or projected lens system.

Fil See H. Dennis Taylor, The Adjustment and Testing of Telescope Objectives, T. Cooke \& Sons (Ltd.); 1921. 
2. Interesting cases in connection with the theory and performance of optical systems can be demonstrated as, for example, the effects of various amounts of different orders of spherical aberration combined with effects of change of focus. The detection of spherical aberration by using infra and extra focal diffraction patterns is also illustrated.

Washington, April 6, 1929. 